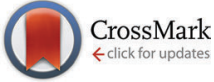

Cite this: Phys. Chem. Chem. Phys., 2016, 18, 9724

Received 5th February 2016 Accepted 10th March 2016 DOI: $10.1039 / c 6 c p 00841 k$

www.rsc.org/pccp

\title{
Tuning of dye optical properties by environmental effects: a $Q M / M M$ and experimental study $\dagger$
}

\author{
Gianluca Del Frate, ${ }^{a}$ Fabio Bellina, ${ }^{\mathrm{b}}$ Giordano Mancini, ${ }^{\mathrm{a}}$ Giulia Marianetti, ${ }^{a}$ \\ Pierpaolo Minei, ${ }^{b}$ Andrea Pucci ${ }^{b}$ and Vincenzo Barone*a
}

\begin{abstract}
The present work is aimed at a deeper investigation of two recently synthesized heteroaromatic fluorophores by means of a computational multilayer approach, integrating quantum mechanics (QM) and molecular mechanics (MM). In particular, dispersion of the title dyes in a polymer matrix is studied in connection with potential applications as photoactive species in luminescent solar concentrators (LSCs). Molecular dynamics simulations, based on accurate QM-derived force fields, reveal increased stiffness of these organic dyes when going from $\mathrm{CHCl}_{3}$ solution to the polymer matrix. QM/MM computations of UV spectra for snapshots extracted from MD simulations show that this different flexibility permits explaining the different spectral shapes obtained experimentally for the two different environments. Moreover, the general spectroscopic trends are reproduced well by static computations employing a polarizable continuum description of environmental effects.
\end{abstract}

\section{Introduction}

Commodity materials based on thermoplastic polymers derived from oil or renewable resources are everyday expanding their range of applications, thanks to their excellent thermo-mechanical properties, chemical stability, easy processing, sustainability and low cost. ${ }^{1}$ A new class of materials is nowadays even more rapidly evolving due to the development of modern technologies that require combinations of properties. This great interest towards functional materials has driven a wide investigation on the design and development of new "intelligent" systems with unique mechanical and optical properties. ${ }^{2-4}$ Several specific features can be finely tuned by an appropriate combination of a polymeric matrix with different molecular entities, by means of dispersion ${ }^{5}$ or covalent functionalization. ${ }^{6,7}$ Such materials can be envisaged as stimuli responsive devices, which can find widespread applications in various fields. ${ }^{8,9}$ Many external factors, such as pressure, temperature, $\mathrm{pH}$, viscosity or radiation, can in fact, alter the system's response. ${ }^{10}$ Owing to these characteristics, optical responsive materials can be exploited in renewable energy technologies; over the last three decades increasing interest has been devoted to light harvesting devices. Recent findings on global warming ${ }^{11}$ and nonrenewable energy

\footnotetext{
${ }^{a}$ Scuola Normale Superiore, Piazza dei Cavalieri 7, I-56126 Pisa, Italy.

E-mail: vincenzo.barone@sns.it

${ }^{b}$ Dipartimento di Chimica e Chimica Industriale, Università di Pisa,

via Moruzzi 13, I-56124 Pisa, Italy

$\dagger$ Electronic supplementary information (ESI) available: (i) Force field parameters for the $\mathbf{a}$ and $\mathbf{b}$ fluorophores and (ii) MD simulation analysis of the $\mathbf{b}$ fluorophore. See DOI: $10.1039 / \mathrm{c} 6 \mathrm{cp} 00841 \mathrm{k}$
}

resources have stimulated increasing interest towards the employment of alternative ways for energy. Sunlight stands indeed as an ideal asset that can be taken advantage of. Luminescent solar concentrators (LSCs) represent a way to decrease the cost of solar photovoltaics. ${ }^{12}$ LSCs consist of a fluorescent dye dispersed in a thin slab of polymeric material. Upon solar irradiation, a fraction of the emitted light is collected at the edges of the device where photovoltaic cells are located. ${ }^{13}$ Organic fluorescent dyes bearing $\pi$-conjugated electron-donor and electron-acceptor moieties exhibit intramolecular charge-transfer (ICT) ${ }^{14}$ properties, and can therefore show the optical properties required by LSCs, such as high quantum yield and large Stokes shift. ${ }^{15}$

Selection of the best molecular structure for such applications is not easily driven by a strategy based on experimental data alone, which can be very approximate. Therefore, computational modeling finds increasing use to carry out systematic studies aimed to gain a deeper understanding of the chemicalphysical properties responsible for the experimental optical features. As a matter of fact, computational approaches nowadays allow simulation of spectral shapes at a very reasonable cost and with good results. ${ }^{16}$ It is therefore not surprising that many and different computational methods, aimed at the reproduction of photophysical properties of organic probes dispersed in - or covalently bounded to - several environments, ${ }^{17-21}$ have been recently reported: such methods, trying to reveal subtle phenomena that take place at the atomic level, hard to detect by means of experimental techniques alone, are becoming an invaluable tool to support experiments. However, the modeling of environmental effects, is often a crucial step, and it needs to 
be taken into account in order to correctly describe the photophysics of a target molecule: charge density can rearrange as a consequence of the electric field generated by the surrounding environment. Moreover, the conformational equilibrium of flexible molecules is often strongly tuned by solvent effects. Unfortunately, it is still arduous to treat the solvent using very accurate quantum mechanical (QM) calculations, because of the computational cost of including a large reservoir of molecules constituting the environment. For these reasons, during the last few decades different approaches have been developed to deal with solvation, ranging from implicit methods, such as the so-called Polarizable Continuum Models (PCM), ${ }^{22}$ to more demanding procedures, such as Monte Carlo (MC), and both classical and $a b$ initio molecular dynamics (MD) simulations, which explicitly feature solvent molecules in their spatial coordinates. ${ }^{23-27}$

In a previous work, ${ }^{28}$ a new class of alkynylimidazole-based fluorophores has been studied as promising luminescent dyes in LSCs. UV-Vis spectra of these molecules in tetrahydrofuran (THF) solution as well as in a poly(methyl methacrylate) (PMMA) thin film were experimentally determined, suggesting an enhanced rigidity of the investigated fluorophores in the polymer matrix. Absorption spectra were simulated in good agreement with experimental data, using the CAM-B3LYP longrange corrected hybrid density functional, in conjunction with the PCM to take bulk environmental effects into account. Moreover, a fast and reliable computational protocol was developed to simulate absorption and emission spectra of such luminescent species when dispersed in the rigid, polymeric environment: starting from the ground-state energy minimum, the main dihedral angle (i.e., the one between the phenyl and the imidazole moieties) was fixed at its ground-state value during the excitation (mimicking in such a way the caging effects of the hydrophobic bundle), while all other internal coordinates have been relaxed. As already stated, bulk electrostatic effects were taken into account by the PCM. As is well known, PCM offers the unquestionable advantage of providing an overall and reliable description of the surrounding environment, which is mainly characterized by its dielectric constant. However, the continuum method fails in the treatment of specific solute-solvent interactions, which depend on the spatial coordinates of the two interacting components. ${ }^{29}$ For this reason, in the present study, a recently developed protocol $^{21}$ was applied to address with improved accuracy the description of the polymeric bundle, and compared to the cheaper "static" approach described above in the reproduction of spectroscopic properties. The proposed approach involves computational methodologies ranging from MD simulations, based on accurate force fields (FF), to full QM calculations, and explicitly simulating the surrounding solvent (the PMMA matrix, in this study). As is well known, FF based simulations manage to mimic the dyes in a realistic environment, and follow the time-evolution of the whole system to its most likely configurations over time scales longer than $100 \mathrm{~ns}$. However, since variations in the chromophore's structure significantly affect the computation of its spectroscopic properties, highly accurate and reliable FF
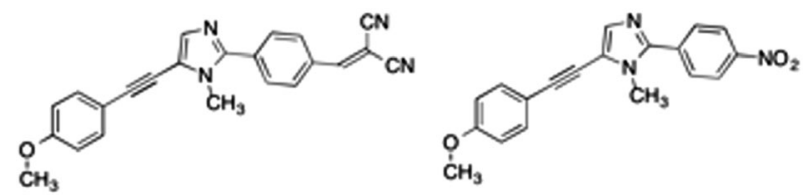

Fig. 1 Structures of the investigated $\mathbf{a}$ (left) and $\mathbf{b}$ (right) alkynylimidazole fluorophores.

parameters are required. For this reason the FF parameterization of the dye used in this work was performed using JOYCE, ${ }^{30,31}$ and all the intra-molecular parameters needed to describe the chromophore in its ground state were specifically derived from QM calculations by fitting optimized energies, gradients and Hessian matrices. Two different alkynylimidazole-based fluorophores bearing a different electron withdrawing group were investigated for their potential application in LSCs, namely the novel 5-((4-dicyanovinyl)ethynyl)-1-methyl-2-(4-nitrophenyl)- $1 H$ imidazole, $\mathbf{a}$, and the already reported ${ }^{25} 5$-((4-methoxy)ethynyl)1-methyl-2-(4-nitrophenyl)-1H-imidazole, b (Fig. 1). Alkynylimidazoles $\mathbf{a}$ and $\mathbf{b}$ were prepared according to a simple reaction sequence, starting from 1-methyl-1 $H$-imidazole.

Evidence for the reduced flexibility of these organic dyes when dispersed in a polymeric bundle is gathered by comparison with chloroform solution, employing classical MD simulations. Then, attention is focused on reproducing absorption spectra in PMMA, and, in particular, on the comparison between different representations of the polymeric environment during such spectroscopic calculations.

\section{Experimental section}

\subsection{Materials}

Melting points were recorded on a hot-stage microscope (Reichert Thermovar). NMR spectra were recorded at room temperature at $400 \mathrm{MHz}\left({ }^{1} \mathrm{H}\right)$ and $100 \mathrm{MHz}\left({ }^{13} \mathrm{C}\right)$ and were referenced to TMS or the residual solvent's signal. All reactions were performed under an argon flow. 1-Methyl- $1 \mathrm{H}$-imidazole was purified by distillation at reduced pressure. $\mathrm{N}$-Bromosuccinimide (NBS) was purified by crystallization from water. All other commercially available reagents and solvents were used as received. 5-((4-Methoxyphenyl)ethynyl)-1-methyl-2-(4-nitrophenyl)-1 $H$-imidazole (b) was prepared and characterized as reported in our previous work. Poly(methylmethacrylate) (PMMA, Aldrich, $M_{\mathrm{w}}=$ $350000 \mathrm{~g} \mathrm{~mol}^{-1}$, acid number $<1 \mathrm{mg} \mathrm{KOH}$ per g) density = $1.17 \mathrm{~g} \mathrm{~mL}^{-1}\left(T=25^{\circ} \mathrm{C}\right)$.

\subsection{4-(1-Methyl-1 $\mathrm{H}$-imidazol-2-yl)benzaldehyde}

A modification of the literature procedure for the Pd- and $\mathrm{Cu}$-mediated $\mathrm{C}-2$ direct arylation of 1-substituted-1 $\mathrm{H}$-imidazole with aryl bromides was adopted. ${ }^{32,33}$ A solution of 1-methyl- $1 H^{-}$ imidazole $(3.75 \mathrm{mmol}, 82.1 \mathrm{mg}, 300 \mu \mathrm{L}), \mathrm{Pd}(\mathrm{OAc})_{2}(28 \mathrm{mg}$, $0.05 \mathrm{mmol}$ ), CuI (952 mg, $5 \mathrm{mmol}$ ), CsOAc (960 mg, $5 \mathrm{mmol}$ ) and 4-bromobenzaldehyde (463 mg, $2.5 \mathrm{mmol})$ in DMA $(15 \mathrm{~mL})$ was stirred at $110{ }^{\circ} \mathrm{C}$ for $72 \mathrm{~h}$. After cooling to room temperature, 
the reaction mixture was diluted with AcOEt $(100 \mathrm{~mL})$ and poured into a saturated aqueous $\mathrm{NH}_{4} \mathrm{Cl}$ solution $(100 \mathrm{~mL}) . \mathrm{NH}_{4} \mathrm{OH}(5 \mathrm{~mL})$ was added and the resulting mixture was stirred in open air for $0.5 \mathrm{~h}$, then extracted with AcOEt $(3 \times 20 \mathrm{~mL})$. The collected organic extracts were washed with water $(2 \times 20 \mathrm{~mL})$, dried over anhydrous $\mathrm{Na}_{2} \mathrm{SO}_{4}$, and concentrated under reduced pressure. The crude reaction product was purified by flash chromatography on silica gel using EtOAc as an eluent to give 4-(1-methyl-1 $H$-imidazol-2-yl)benzaldehyde as a yellow solid (206 mg, 44\% yield): m.p. $43-44{ }^{\circ} \mathrm{C}$. ${ }^{1} \mathrm{H}$ NMR (400 MHz, $\left.\mathrm{CDCl}_{3}\right): \delta=10.04(\mathrm{~s}, 1 \mathrm{H}), 7.95(\mathrm{~m}, 2 \mathrm{H}), 7.82$ $(\mathrm{m}, 2 \mathrm{H}), 7.16$ (d, $J=1.2 \mathrm{~Hz}, 1 \mathrm{H}), 7.02(\mathrm{~d}, J=1.2 \mathrm{~Hz}, 1 \mathrm{H}), 3.80(\mathrm{~s}, 3 \mathrm{H})$. ${ }^{13} \mathrm{C} \mathrm{NMR}\left(100 \mathrm{MHz}, \mathrm{CDCl}_{3}\right): \delta=191.7,146.3,136.2,135.9,129.1$ (2C), 128.9, 128.8 (2C), 123.3, 34.8 ppm. MS (EI) m/z (\%): 187 (12), 186 (100), 185 (95), 157 (23), 156 (20).

\subsection{4-(5-((4-Methoxyphenyl)ethynyl)-1-methyl-1 $H$-imidazol-2-yl)- benzaldehyde}

According to our recently reported procedure, ${ }^{34}$ NBS (140 mg, $0.39 \mathrm{mmol}$ ) was added in one portion to a solution of 4-(1-methyl- $1 H$-imidazol-2-yl)benzaldehyde (142 mg, $0.8 \mathrm{mmol}$ ) in DMF $(8 \mathrm{~mL})$, and the resulting mixture was stirred in the dark at room temperature for $12 \mathrm{~h}$. Then, $\mathrm{Pd}\left(\mathrm{PPh}_{3}\right)_{2} \mathrm{Cl}_{2}(12 \mathrm{mg}$, $0.016 \mathrm{mmol}, 2 \mathrm{~mol} \%$ ), CuI (6 mg, $0.032 \mathrm{mmol}, 4 \mathrm{~mol} \%$ ), 4-ethynylanisole $(200 \mu \mathrm{L}, 1.5 \mathrm{mmol})$, and piperidine $(240 \mu \mathrm{L}$, $2.4 \mathrm{mmol}$ ) were sequentially added under a stream of argon. The resulting reaction mixture was stirred at $80{ }^{\circ} \mathrm{C}$ for $4 \mathrm{~h}$, then cooled to room temperature and treated with EtOAc $(50 \mathrm{~mL})$ and saturated aqueous $\mathrm{NH}_{4} \mathrm{Cl}(50 \mathrm{~mL})$. The resulting biphasic mixture was stirred for 30 minutes in open air, then extracted with EtOAc $(3 \times 10 \mathrm{~mL})$. The organic extracts were collected, washed with water $(3 \times 10 \mathrm{~mL})$ and brine $(10 \mathrm{~mL})$, dried over anhydrous $\mathrm{Na}_{2} \mathrm{SO}_{4}$, filtered and concentrated under reduced pressure. The crude reaction product was purified by flash chromatography on silica gel using a mixture of toluene and AcOEt (6/4) as an eluent, to give the title compound as a pale orange solid (125 mg, 52\% yield): m.p. $119-121{ }^{\circ} \mathrm{C} .{ }^{1} \mathrm{H}$ NMR $\left(400 \mathrm{MHz}, \mathrm{CDCl}_{3}\right): \delta=10.10(\mathrm{~s}, 1 \mathrm{H}), 8.02(\mathrm{~m}, 2 \mathrm{H}), 7.91(\mathrm{~m}, 2 \mathrm{H})$, $7.49(\mathrm{~m}, 2 \mathrm{H}), 7.47$ (s, 1H) ppm, $6.93(\mathrm{~m}, 2 \mathrm{H}), 3.89$ (s, 3H), 3.87 $(\mathrm{s}, 3 \mathrm{H}) .{ }^{13} \mathrm{C}$ NMR $\left(100 \mathrm{MHz}, \mathrm{CDCl}_{3}\right): \delta=191.7,160.3,146.8$, 136.4, 135.8, 134.1, 132.9 (2C), 130.2 (2C), 128.7 (2C), 119.3, 114.6, 114.0 (2C), 97.5, 76.2, 55.4, $33.3 \mathrm{ppm}$. MS (EI) $\mathrm{m} / \mathrm{z}(\%)$ 317 (23), 316 (100), 315 (29), 207 (10).

\subsection{5-((4-Methoxyphenyl)ethynyl)-1-methyl-2-(4-dicyanovinyl- phenyl)-1H-imidazole (a)}

According to a literature procedure, ${ }^{35}$ 4-(5-((4-ethoxyphenyl)ethynyl)-1-methyl-1 $H$-imidazol-2-yl)benzaldehyde ( $64 \mathrm{mg}, 0.2 \mathrm{mmol}$ ) was reacted with malononitrile $(20 \mathrm{mg}, 0.3 \mathrm{mmol})$ in EtOH $(7 \mathrm{~mL})$ and a catalytic amount (5 drops) of triethylamine. The reaction mixture was stirred at room temperature for $12 \mathrm{~h}$, then concentrated under reduced pressure to obtain the required product as a bright orange powder (60 mg, 83\% yield): m.p. $158-159{ }^{\circ} \mathrm{C}$. ${ }^{1} \mathrm{H}$ NMR (400 MHz, $\left.\mathrm{CDCl}_{3}\right): \delta=8.04(\mathrm{~m}, 2 \mathrm{H}), 7.93(\mathrm{~m}, 2 \mathrm{H}), 7.82$ (s, 1H), $7.50(\mathrm{~m}, 2 \mathrm{H}), 7.48(\mathrm{~s}, 1 \mathrm{H}), 6.93(\mathrm{~m}, 2 \mathrm{H}), 3.92(\mathrm{~s}, 3 \mathrm{H}), 3.87$ (s, 3H). ${ }^{13} \mathrm{C} \mathrm{NMR}\left(100 \mathrm{MHz}, \mathrm{CDCl}_{3}\right): \delta=160.2,158.8,146.0,134.3$, 133.0 (2C), 131.1 (2C), 130.7, 129.0 (2C), 120.0, 114.2 (2C), 113.7
(2C), 112.6, 108.8, 97.9, 83.0, 75.9, 55.4, 33.4 ppm. MS (EI) $m / z(\%):$ 365 (30), 364 (100), 349 (23), 281 (38), 208 (18), 207 (61).

\subsection{Preparation of polymer films for optical studies}

Different dye/PMMA thin films were prepared by drop casting, i.e. pouring a $0.8 \mathrm{~mL}$ chloroform solution containing $30 \mathrm{mg}$ of the polymer and the proper amount of dye to obtain concentrations in the range of $0.05-2.2 \mathrm{wt} \%$ over a $35 \times 50 \mathrm{~mm}$ area of a glass surface. The glass slides were cleaned with chloroform and immerged in $6 \mathrm{M} \mathrm{HCl}$ for at least $12 \mathrm{~h}$, then they were rinsed with water, acetone and isopropanol and dried for $8 \mathrm{~h}$ at $120{ }^{\circ} \mathrm{C}$. Solvent evaporation was performed on a warm hot plate (about $30{ }^{\circ} \mathrm{C}$ ) and in a closed environment. The film thickness was measured by a Starrett micrometer to be $25 \pm 5$ microns.

\section{Computational details}

\subsection{General approach}

The applied computational protocol (sketched in Fig. 2), already tested in previous studies, ${ }^{21,23}$ can be summarized as follows:

(1) QM calculations are performed to sample the conformational space of the considered dyes to find their global minimum. Energies, first and second derivatives, are computed, together with $\mathrm{CM} 5^{36}$ point charges. Next, partial geometry optimizations are performed, constraining specific internal coordinates at selected values, in order to describe soft degrees of freedom (i.e., flexible dihedrals). The obtained data are then used to optimize FF parameters.

(2) Reliable models for both PMMA and $\mathrm{CHCl}_{3}$ are built, by means of MM methods using FF parameters taken from the literature. $\mathrm{MD}$ simulation of the fluorophores in the two different considered environments (i.e., in the real sized polymer matrix and in $\mathrm{CHCl}_{3}$ solution) are then performed and analyzed.

(3) Sufficient number of statistically uncorrelated snapshots is extracted from the MD trajectories, once the inspection of the internal motions of the dyes indicates that the accessible conformations have been sampled with sufficient accuracy.

(4) Absorption spectra are finally computed and compared on one hand with experiments and on the other hand with the results obtained using a polarizable continuum representation of the environment (i.e., the polymeric matrix).

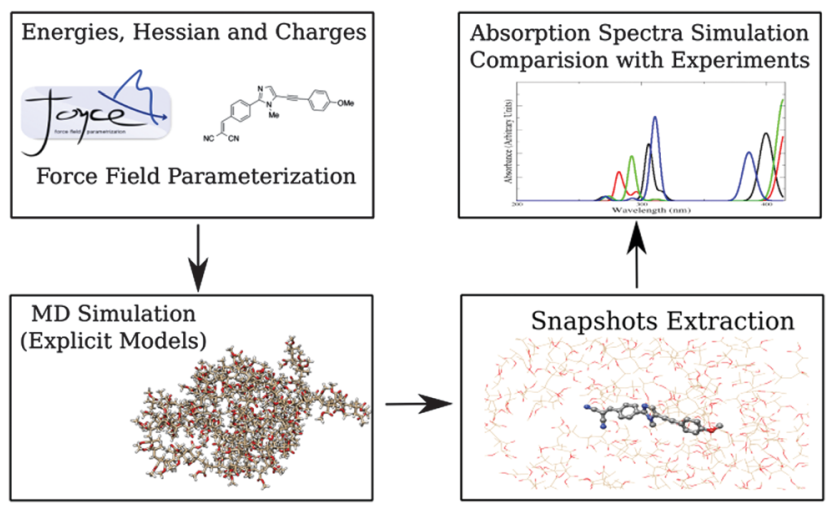

Fig. 2 Sketch of the multilevel protocol employed in the present work. 


\subsection{QM calculations}

QM calculations were performed to (i) obtain reference structures, for parameterizing classical FFs, and to (ii) compute absorption spectra.

For both compounds a and $\mathbf{b}$, global energy minima were located at the DFT level, using the B3LYP exchange-correlation functional and the SNSD basis set. ${ }^{37,38}$ To reproduce experimental conditions, solvent effects were taken into account by means of the conductor-like variant of PCM (C-PCM). ${ }^{39}$ In particular, butanoic acid was considered as the solvent, because of its dielectric constant (2.9931) similar to that of PMMA (3-3.3). Moreover, the Hessian matrix and harmonic vibrational frequencies were evaluated on the computed global minimum. In order to describe flexible terms in the fluorophores FFs and QM energy scans along flexible dihedrals were performed, all the other geometrical parameters being optimized at fixed values of the soft variable spaced by $30^{\circ}$. The structures obtained from those relaxed scans were then used by JOYCE software in order to fit dihedral FF parameters. Absorption spectra were computed for several (roughly, two-hundred for compound) statistically uncorrelated snapshots, extracted from MD simulations running in the polymeric environment. Environmental effects were taken into account by the so-called electrostatic embedding (EE) ${ }^{40,41}$ in which all the retained PMMA atoms are represented by their OPLS $^{42}$ point charges. At larger distances, bulk solvent effects were accounted for by means of the PCM. Electronic transitions were computed using the TD-DFT method, and considering the six lowest electronic states for each snapshot. The CAM-B3LYP functional and the SNSD basis set were used: this combination, in fact, has already been shown to be suitable for these kinds of systems.

All the QM calculations were performed using the Gaussian 09 suite of programs. ${ }^{43}$

\subsection{Force field parameterizations}

As stated above, JOYCE software was used to obtain accurate intra-molecular FFs for $\mathbf{a}$ and $\mathbf{b}$. The analytical form, $E_{\text {intra, }}$, includes the standard bonded and non-bonded contributions, namely:

$$
E_{\text {intra }}=E_{\text {stretch }}+E_{\text {bend }}+E_{\text {Rtorsion }}+E_{\text {ftorsion }}+E_{\mathrm{nb}}
$$

The first three terms describe the contribution of "stiff" terms (i.e., bonds, angles and rigid dihedrals) to the overall intramolecular energy. Such terms are, respectively, described using the following harmonic forms:

$$
\begin{aligned}
E_{\text {stretch }} & =\frac{1}{2} \sum_{\mu}^{N_{\text {bonds }}} k_{\mu}^{\mathrm{s}}\left(b_{\mu}-b_{\mu}^{0}\right)^{2} \\
E_{\text {bending }} & =\frac{1}{2} \sum_{\mu}^{N_{\text {angles }}} k_{\mu}^{\mathrm{b}}\left(\theta_{\mu}-\theta_{\mu}^{0}\right)^{2} \\
E_{\text {Rtors }} & =\frac{1}{2} \sum_{\mu}^{N_{\text {Rtors }}} k_{\mu}^{\mathrm{t}}\left(\phi_{\mu}-\phi_{\mu}^{0}\right)^{2}
\end{aligned}
$$

where $k_{\mu}^{\mathrm{s}}, k_{\mu}^{\mathrm{b}}$, and $k_{\mu}^{\mathrm{t}}$ and $b_{\mu}^{0}, \theta_{\mu}^{0}$, and $\phi_{\mu}^{0}$ are, respectively, the force constants and equilibrium values for stretching, bending and stiff torsions.

The potential around flexible dihedrals (Ftors) is usually described by the leading terms of a Fourier series:

$$
E_{\mathrm{Ftors}}=\sum_{\mu}^{N_{\mathrm{Ftors}}} \sum_{j}^{N_{\mu}} k_{\mu j}^{\mathrm{d}}\left[1+\cos \left(n_{j}^{\mu} \delta_{\mu}-\gamma_{j}^{\mu}\right)\right]
$$

Here, $N_{\mu}$ is the number of cosine functions used to describe the considered $\mu$ dihedral, while $\mathrm{n}_{\mu}^{j}$ and $\gamma_{\mu}^{j}$ are the multiplicity and the phase factor of the $j$-th cosine term.

The last term in eqn (1) represents non-bonded interactions, consisting of Lennard-Jones and Coulomb contributions, i.e.

$$
E_{\mathrm{nb}}=\sum_{i}^{N_{\mathrm{nb}}} \sum_{j>i}^{N_{\mathrm{nb}}} 4 \varepsilon_{i j}^{\mathrm{intra}}\left[\left(\frac{\sigma_{i j}^{\mathrm{intra}}}{r_{i j}}\right)^{12}-\left(\frac{\sigma_{i j}^{\mathrm{intra}}}{r_{i j}}\right)^{6}\right]+\sum_{i}^{N_{\mathrm{nb}}} \sum_{j>i}^{N_{\mathrm{nb}}} \frac{q_{i} q_{j}}{r_{i j}}
$$

where $\varepsilon_{i j}^{\text {intra }}$ is the well depth, $\sigma_{i j}^{\text {intra }}$ is the sphere radius, $q_{i}$ is the charge on atom $i$ and $r_{\mathrm{ij}}$ is the distance between the two considered atoms $i$ and $j$.

FF parameters are obtained using JOYCE through the minimization of the following merit function $I_{\mathrm{g}}$ :

$$
\begin{aligned}
I_{g}= & \sum_{g=0}^{N_{\text {geom }}} W_{g}\left[U_{g}-E_{g}^{\text {intra }}\right]^{2} \\
& +\sum_{K \leq L}^{3 N-6} \frac{2 W_{K L}^{\prime \prime}}{(3 N-6)(3 N-5)}\left[H_{K L}-\left(\frac{\partial^{2} E^{\text {intra }}}{\partial Q_{K} \partial Q_{L}}\right)\right]_{g=0}^{2}
\end{aligned}
$$

Here, $N_{\text {geom }}$ accounts for the considered molecular geometries, $Q_{K}$ is the $K$-th Cartesian coordinate and $U_{g}$ is the QM energy difference between the $g$-th geometry and the global minimum (i.e., $g=0$ ). The QM Hessian matrix and the corresponding FF Hessian are both computed at $g=0 . W_{g}$ and $W^{\prime \prime}$ are weighting factors. More information about the JOYCE parameterization procedure can be found in previous studies. ${ }^{30,31}$

For all the considered dyes, LJ parameters were transferred directly from the OPLS FF and atomic charges were set to their CM5 values computed at the global energy minimum. The OPLS FF was used to describe the PMMA polymer matrix, and the general Amber FF (GAFF) ${ }^{44}$ for $\mathrm{CHCl}_{3}$.

\subsection{Molecular modeling and MD simulations}

A random sequence of atactic PMMA, in a completely linear conformation, was built by using an in-house Python (www. python.org) script, thus obtaining a real-sized macromolecule of approximately $300 \mathrm{kDa}$ (therefore, having 2920 monomers of methacrylic acid). Such conformation was then minimized using the conjugate gradient algorithm, until an energy threshold of $0.5 \mathrm{~kJ} \mathrm{~mol}^{-1}$ was reached. A replica of this minimized conformation was added and rotated, with the aim of increasing the complexity of the system. Preliminary MD runs were performed in vacuo, to equilibrate the polymer: the simulation time-step was initially set to 0.1 fs for the first 200 ps and then increased to 0.5 fs for the remaining 4.8 ns. A $14 \AA$ cutoff was 
applied for both the electrostatic and the van der Waals (vdW) interactions. When a curled conformation was eventually reached by the polymer, periodic boundary conditions were applied in all the directions and the dye was manually introduced inside the polymer matrix and kept frozen at its equilibrium conformation. The system was coupled to a thermal bath at $300 \mathrm{~K}$ and to a pressure bath at 1 bar through weak coupling schemes, ${ }^{45}$ thus running in the NPT ensemble. Coupling constants were set to $0.1 \mathrm{ps}$ and $0.5 \mathrm{ps}$, respectively. vdW forces were computed applying a cutoff distance of $13 \AA$, whereas long-range electrostatic interactions were treated with the particle mesh Ewald (PME) method. After 20 ns of MD simulation, the final density was found to be lower than $1.00 \mathrm{~g} \mathrm{~cm}^{-3}$, pretty far from the average experimental value (1.17-1.19 $\left.\mathrm{g} \mathrm{cm}^{-3}\right)$. To achieve a mass density closer to the experimental range, all the angles' force constant values were reduced by $50 \%$. Moreover, the coupling constant to the barostat was reduced from 0.5 to $0.1 \mathrm{ps}$. Under these conditions, after $20 \mathrm{~ns}$ of simulation, a density value of $1.20 \mathrm{~g} \mathrm{~cm}^{-3}$ was obtained. Such collapsed structure was finally re-equilibrated by restoring the system under the initial conditions. After $5 \mathrm{~ns}$, the density value was detected to be stable at $1150 \mathrm{~kg} \mathrm{~m}^{-3}$ (a reasonable value taking into account the complexity of the simulated system).

The starting coordinates for the liquid chloroform box setup were taken from the coordinate file available on the Virtual Chemistry server. ${ }^{46}$ CM5 charges were computed on the $\mathrm{CHCl}_{3}$ molecule, modeling the environment $\left(\mathrm{CHCl}_{3}\right)$ with PCM. The box size was tripled, passing from 1000 to $3174 \mathrm{CHCl}_{3}$ molecules. The LINCS constraining algorithm ${ }^{47}$ was applied to all the chemical bonds. A first equilibration in the NPT ensemble of $2.0 \mathrm{~ns}$ was performed, using a time-step of $0.2 \mathrm{fs}$ for the first $500 \mathrm{ps}$ and $2.0 \mathrm{fs}$ for the last $1.5 \mathrm{~ns}$.

Two sets of MD simulations were performed: on the solvated and the polymer-embedded dye. Thus, to study dyes a and $\mathbf{b}, \mathbf{a}$ total of four MD simulations were performed. For the polymer matrix, MD was carried out in the NPT ensemble to match the experimental conditions, and using the same conditions already mentioned for the PMMA modeling step. The last snapshot coming from the PMMA-density equilibration was used as the starting conformation. The fluorophore was "unfrozen", and the total sampling time was set to 50 ns. The timestep was set to 2 fs and all the bonds were constrained at their initial value by means of the LINCS algorithm. System coordinates were saved every ps. The simulations in the chloroform solution were performed in the NVT ensemble, at $300 \mathrm{~K}$. The total sampling time was set again to $50 \mathrm{~ns}$. All the chemical bonds of the dye and the solvent were constrained.

All the simulations described above were performed using GROMACS 4.6.5. ${ }^{48}$

\subsection{Absorption spectra calculations}

Transition energies obtained for the MD snapshots were broadened by means of Gaussian functions, using a half width at half maximum (HWHM, and indicated as $\Delta_{\mathrm{v}}$ in eqn (6)) of $0.25 \mathrm{eV}$. This value was chosen to match experimental data.
For a generic snapshot c, the spectrum is then computed as

$$
\varepsilon_{\mathrm{c}}(v) \propto \sum_{\mathrm{i} \in \operatorname{states}_{\mathrm{c}}} \frac{f_{\mathrm{c}, \mathrm{i}}}{\Delta_{\mathrm{v}}} \exp \left[\left(\frac{v-v_{\mathrm{c}, \mathrm{i}}^{0}}{\sigma_{\mathrm{v}}}\right)^{2}\right]
$$

where $\sigma_{\mathrm{v}}=[2 \sqrt{2 \ln 2}]^{-1} \cdot \Delta_{\mathrm{v}}, v_{\mathrm{c}, \mathrm{i}}^{0}$ and $f_{\mathrm{c}, \mathrm{i}}$ are the excitation energy and the dimensionless oscillator strength of the considered $i$ excitation, respectively. For an appropriate comparison with the experiments, the obtained spectra are plotted in the wavelength domain $\left(\varepsilon_{\mathrm{c}}(\lambda)\right)$. The final absorption spectrum is obtained by averaging the individual signals, according to

$$
\bar{\varepsilon}(\lambda)=\sum_{\mathrm{c} \in \text { snap }} \frac{\varepsilon_{\mathrm{c}}(\lambda)}{N_{\text {snaps }}}
$$

\section{Results}

\subsection{Fluorophore force fields}

As indicated in Section 3.3, van der Waals (vdW) parameters were directly transferred from published force fields. For both a and b, CM5 charges were computed at geometries optimized in butanoic acid at the DFT/PCM level.

No strong differences were found on the two minima, as shown in Fig. 3, where the $\mathbf{a}$ and $\mathbf{b}$ minima structures are superimposed. In particular, values of $28^{\circ}$ and $30^{\circ}$ for the dihedral angle between the imidazole and the pull-phenyl was found for $\mathbf{a}$ and $\mathbf{b}$, respectively. Starting from a, and as already mentioned in Section 3.3, the parameterization was performed through the minimization of eqn (5) for each scanned internal coordinate, obtaining an overall standard deviation of $0.00624 \mathrm{~kJ} \mathrm{~mol}^{-1}$. The same equilibrium geometrical parameters and force constants were used for equivalent internal coordinates only if contained within the same molecular block, e.g. the phenyl-pull or the phenyl-push moiety. The flexibility of the molecule under examination does not permit the use of only the absolute energy minimum to obtain a reliable FF. Therefore, a reference QM scan was performed for all the dihedral angles that could affect the overall conformation (and indicated in Fig. 4 as $\delta_{1-5}$ ), using the procedure already described in the section devoted to computational details. The obtained energy profiles for the five dihedral angles are shown in Fig. 4, where single point energies calculated at the QM level are compared to their FF counterparts.

The good agreement between both levels of theory (see Fig. 4) points out that our FF performs a remarkable job in reproducing the structures and relative energies of different conformers.

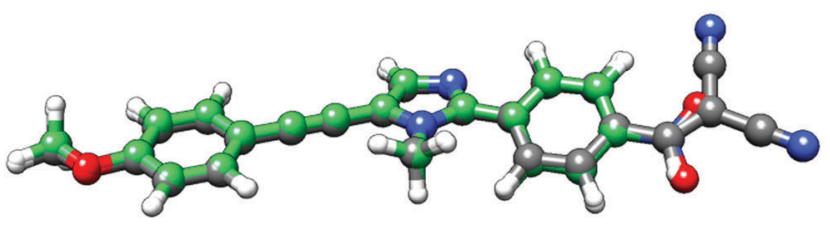

Fig. 3 Superposition of $\mathbf{a}$ (carbons in grey) and $\mathbf{b}$ (carbons in green) computed minima. 


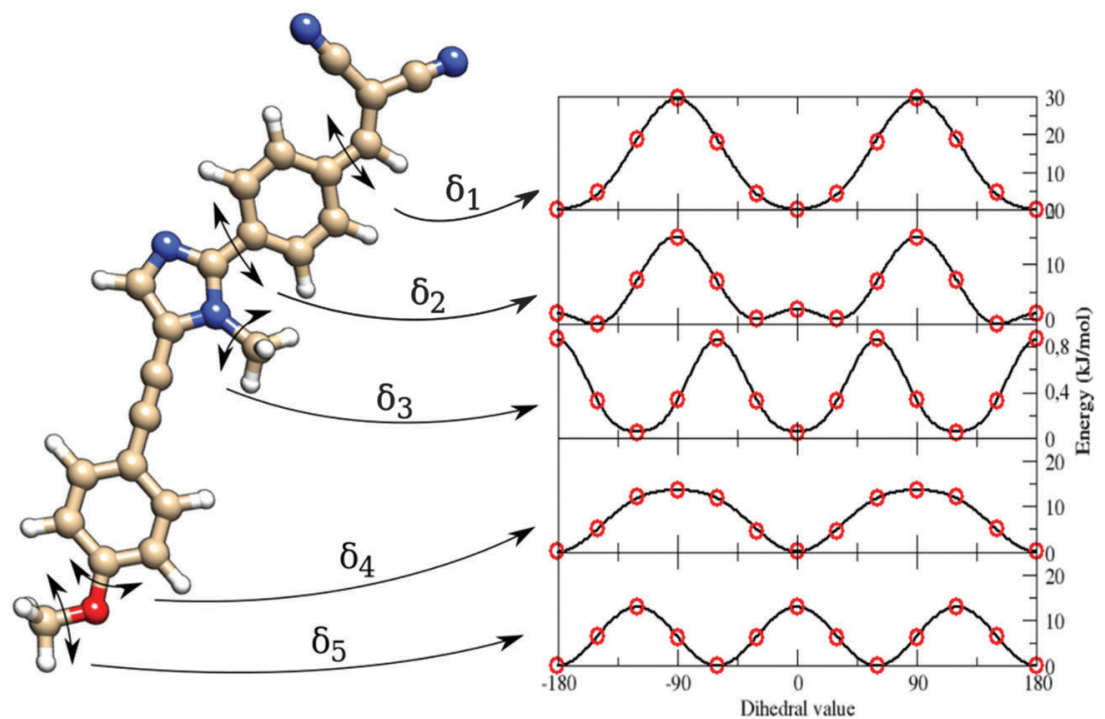

Fig. 4 Left: Structure of the dye a. Right: Energy profiles along the five parameterized flexible dihedrals (from $\delta_{1}$, on top, to $\delta_{5}$, on bottom) at the QM (red circles) and the Joyce FF (continuous line) levels.

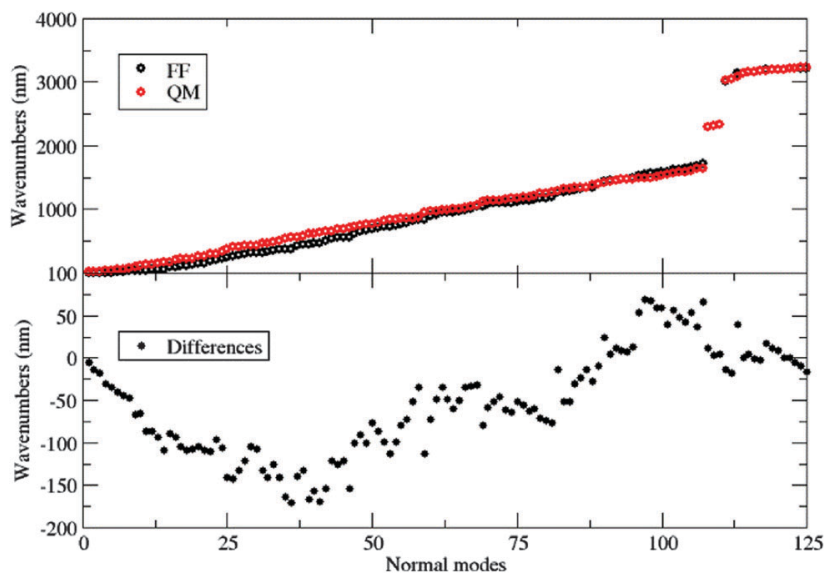

Fig. 5 Top: Computed vibrational frequencies (both at the QM and FF levels) of fluorophore $\mathbf{a}$. Bottom: Differences between the two descriptions.

All the potential energy curves along the five torsional angles are symmetric about the origin. The $\delta_{1}$ and $\delta_{4}$ dihedral angles show energy minima at $0^{\circ}$ and $180^{\circ}$, i.e. for planar arrangements of the whole molecule. The largest geometry variations are related to the $\delta_{2}$ dihedral angle, which shows four different energy minima at $\pm 30^{\circ}$ and $\pm 150^{\circ}$. However, planar conformers $\left(0^{\circ}\right.$ and $\left.180^{\circ}\right)$ are also quite stable due to the significant electron delocalization between the two connected rings. The capability of the FF of a to reproduce the vibrational behavior of the molecule was checked by comparing QM and FF harmonic frequencies. Fig. 5 shows that the QM trend is well reproduced by the FF calculations, with a root-mean square deviation of $82 \mathrm{~cm}^{-1}$, which can be considered satisfactory for the purposes of the present study.

With the aim of developing a FF also for the $\mathbf{b}$ dye, a somewhat different strategy was used: FF parameters for the common, planar structure were simply transferred from a to $\mathbf{b}$. Then, reference geometrical parameters and force constants
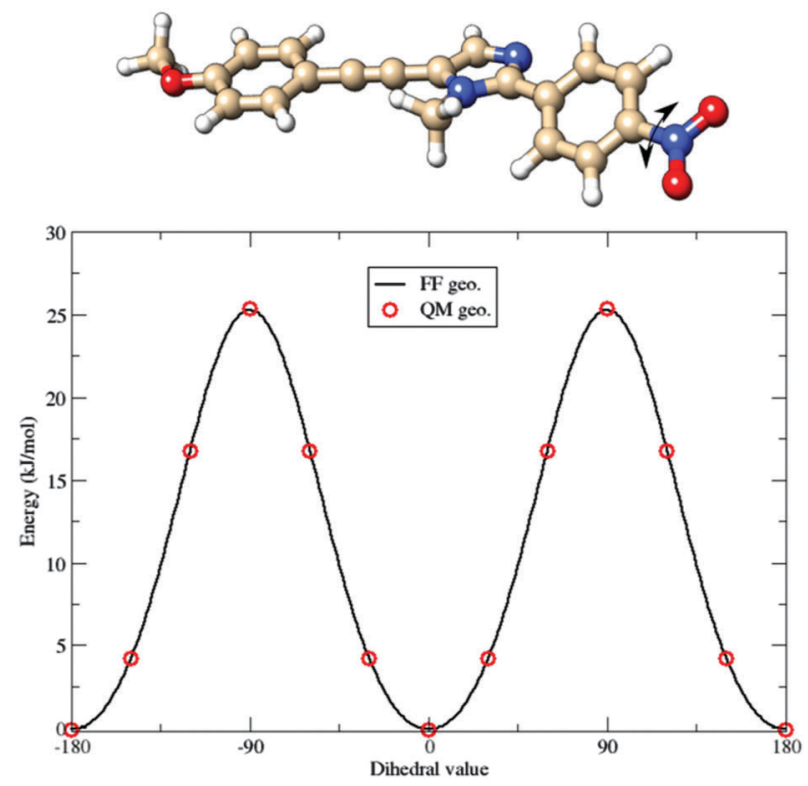

Fig. 6 Comparison between QM (red circles) and Joyce FF (continuous line) potential energy curves governing the torsion of the nitro group in the b fluorophore.

relative to the nitro group were computed for the $\mathbf{b}$ molecule, freezing all the other parameters at the values optimized for $\mathbf{a}$. The results related to the parameterization of the torsion of the nitro group are shown in Fig. 6. The full FF parameter set can be found in the ESI. $\dagger$

\subsection{MD simulation analysis}

The enhanced rigidity of the fluorophores upon dispersion in the polymer bundle was postulated in ref. 28 to explain the reduced efficiency of quenching effects. In fact, quantum yields determined in solution and PMMA have essentially the same 


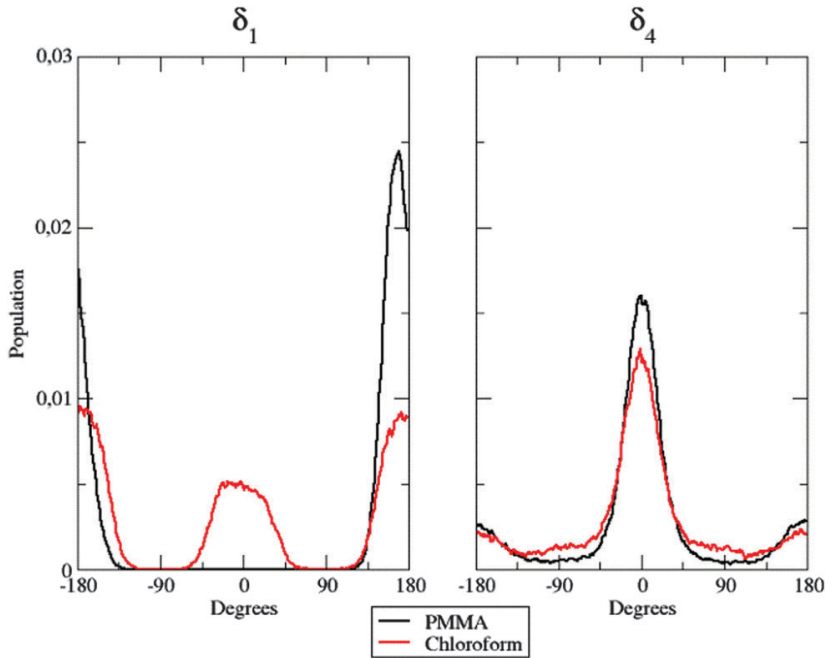

Fig. 7 Population distribution of the $\delta_{1}$ (left panel) and the $\delta_{4}$ (right panel) flexible dihedrals in the two considered environments.

value $(\approx 0.1)$ : while this parameter in solution is widely affected by large conformational changes that take place between the ground and the excited states, in the polymer matrix these structural conversions are not allowed. The goal of the MD simulations is therefore two-fold: on the one hand they permit the generation of statistically uncorrelated snapshots on which spectroscopic properties can be computed and, on the other hand, they also permit demonstrating, at an atomistic level, the reduced flexibility of the dispersed molecules, due to polymer caging effects. In the following we will explicitly consider only the dye $\mathbf{a}$, whereas the results of dye $\mathbf{b}$ are given in the ESI. $\dagger$

Interesting aspects are evidenced by inspection of Fig. 7 .

In the left panel, it is easy to note differences among the behavior of the dye when dispersed in the polymer matrix and in solution: in the former case, $\delta_{1}$ is frozen at approximately $180^{\circ}$, whereas both planar conformations $\left(0^{\circ}\right.$ and $\left.180^{\circ}\right)$ are populated in the latter case. This fact could be due to the hindering effect exhibited by the polymer chains: their steric hindrance prevents the dye from any conformational rearrangement along the considered dihedral, since such torsion implies the movement of a large and bulky group (the dicyanovinyl moiety). Concerning next $\delta_{4}$ (right panel in Fig. 7), no significant differences were found between the two different environments: nonetheless, in PMMA, the population is more peaked at $0^{\circ}$ than in chloroform solution, highlighting again the constraining effect of the polymer matrix. This trend is confirmed by the behavior of $\delta_{2}$.

As a matter of fact, the inter-conversion between the allowed conformations of $\delta_{2}$ is much faster in $\mathrm{CHCl}_{3}$ than inside the polymer (Fig. 8, left panel): indeed, during the considered $50 \mathrm{~ns}$, only two well-defined transitions from one conformer to the other were observed, the first one occurring after more than 15 ns.

Furthermore, in $\mathrm{CHCl}_{3}$, the four different conformers of the dye show comparable populations. In contrast, it is clear that $50 \mathrm{~ns}$ are not sufficient to obtain a fully equilibrated population of all the energy minima when the dye is within the PMMA thin

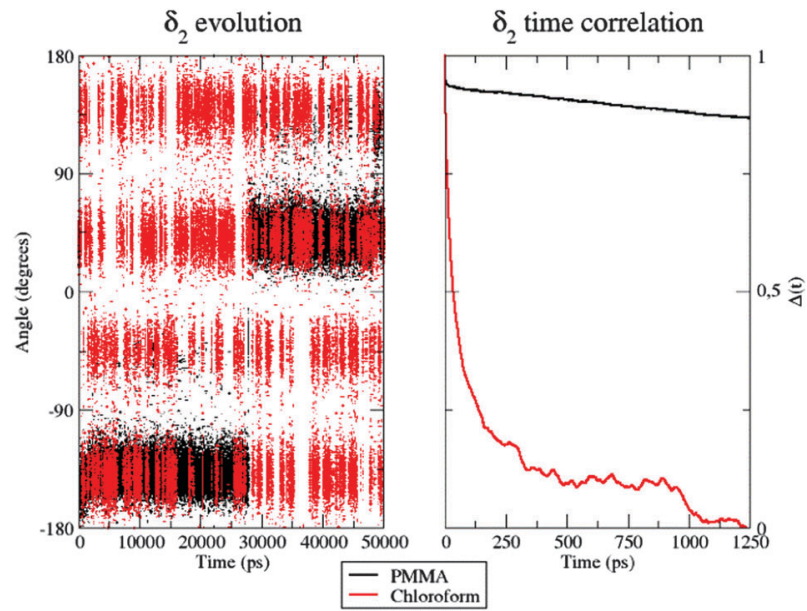

Fig. 8 Left: Time evolution of the $\delta_{2}$ dihedral in the PMMA matrix (black) and chloroform solution (red). Right: Time correlation of the $\delta_{2}$ dihedral in the two environments.

film, because of the very slow structural conversions allowed by the polymeric entanglement. This observation was also confirmed by looking at the $\delta_{2}$ time autocorrelation function (ACF), computed as

$$
\Delta(t)=\cos \left[\delta_{2}\left(t_{0}+t\right)\right] \cos \left[\delta_{2}\left(t_{0}\right)\right]
$$

and shown in the right panel of Fig. 8. This is apparent from the trend of the fluorophore to assume different conformations along $\delta_{2}$ in chloroform solution, with the $\Delta(t)$ value approaching zero after only one ns of simulation. Conversely, the same dihedral appears to be constant, remaining highly correlated for a much longer time, when the dye is within the polymer environment.

Further evidence of different flexibilities of the investigated dye among the PMMA chains and the chloroform solutions was provided by the analysis of other structural features, such as (i) the radius of gyration and (ii) the distance between the pulland the push-phenyl rings, placed at the ends of the dye. Such parameters give an overall description of the internal mobility of the fluorophore in the medium under examination. The radius of gyration is well known to provide a rough measure of the compactness of a structure, and has been calculated as

$$
R_{\mathrm{g}}=\sqrt{\frac{\sum_{i} r_{i}^{2} m_{i}}{\sum_{i} m_{i}}}
$$

where $m_{i}$ is the mass of atom $i$ and $r_{i}$ is the position of atom $i$ with respect to the center of mass of the molecule. The normalized trend is plotted in Fig. 9, left panel.

The hardness encountered by the dye is evident in the polymer simulation, where the radius of gyration is stable at $0.6 \mathrm{~nm}$. Similar conclusions can be drawn by looking at the evolution of the distance between the push- and the pullmoieties during the simulation. As shown in Fig. 9, right panel, the polymer does not allow the dispersed molecule to relax along its major axis, thus keeping the distance between the two 


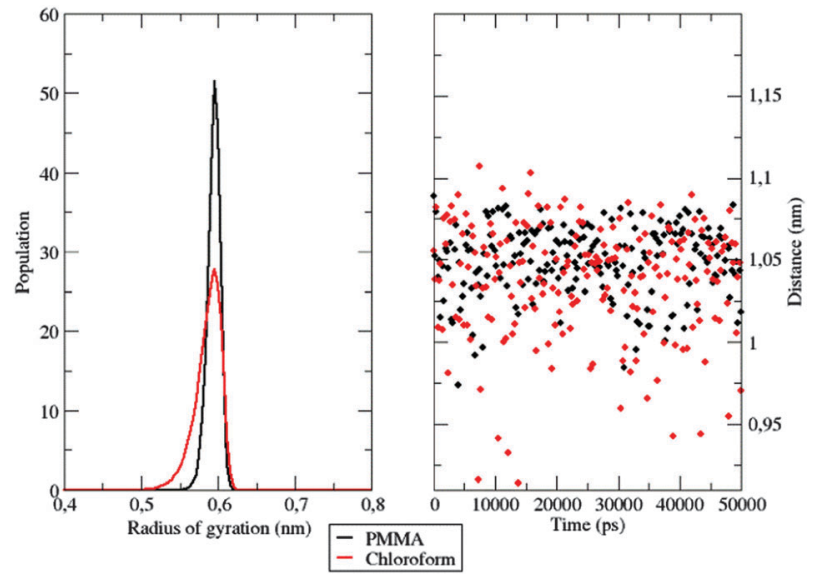

Fig. 9 Left: Normalized gyration radius distribution of the investigated dye among the sampled time in the two different simulations. Right: Distance evolution between the two phenyl rings.

considered groups around $1.05 \mathrm{~nm}$ for the whole time span. In contrast, in the chloroform solution, the distance between the two considered groups oscillates frequently during the simulation, thanks to the higher flexibility of the surrounding environment.

The computation of dynamic properties in the two different media could permit gaining further insights also concerning the effects of the considered environments on the fluorophore's dynamics. The mean square displacement (MSD) of the center of mass of a was computed for both simulations as

$$
\mathrm{MSD}=\lim _{t \rightarrow \infty} \frac{1}{6 t}\left[r\left(t_{0}\right)-r\left(t_{0}+t\right)\right]^{2}
$$

where $r$ has been considered as the center of mass position of the molecule.

Fig. 10 shows the MSD variation during the first 50 ps.

The behavior of the dye in chloroform is typical for a molecular solute in a liquid solvent, where a diffusive regime is quickly approached. In contrast, in the polymer matrix, the MSD value suddenly reaches a plateau: the solute is trapped, its

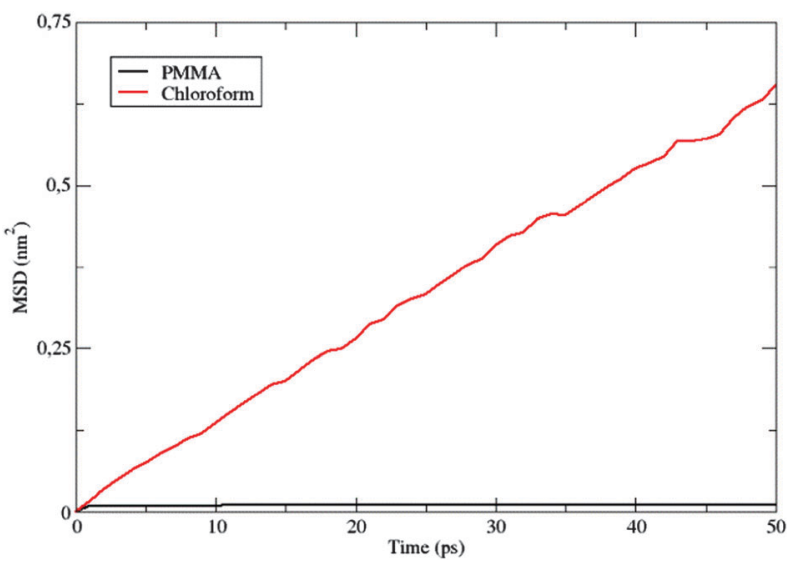

Fig. 10 Comparison between the $\operatorname{MSD}(t)$ values computed in the PMMA matrix and chloroform solution. motion being strongly hindered by the surrounding PMMA cage, which delays the establishment of a diffusive regime. Moreover, the absence of a sub-diffusive behavior indicates that the polymer chains are also not diffusing, i.e. the polymer behaves as a plastic, thin film.

\subsection{UV absorption spectra}

All the results analyzed in Section 4.2 point out a strong rigidity of the dyes induced by the caging effect of the polymeric matrix. It is, therefore, natural to assume that structural changes between the ground and excited electronic states are severely restricted in the polymer bundle. Keeping this in mind, it seems reasonable to simulate polymer hindering effects by the previously adopted ${ }^{28}$ static approach, where the $\delta_{2}$ dihedral angle is fixed to its ground-state value and bulk polymer effects are taken into account by the PCM. MD simulations permit checking this simplified model by looking at the populations of the different conformers along the time. The results (see Section 4.2, Fig. 8 - left panel) clearly show that not all the low energy conformers are equally populated. Interconversions ruled by the $\delta_{2}$ dihedral are very slow, because of the caging effect of the polymer, so that $50 \mathrm{~ns}$ are not sufficient to reach equilibrium values of these populations. In order to obtain a more homogeneous collection of conformers for computing equilibrium spectroscopic properties, one additional MD simulation was performed: in this case the starting configuration of the considered dye was symmetrized along the $\delta_{2}$ dihedral angle. Then, absorption spectra were computed, in the case of the dye within the polymer, by averaging single signals, computed at the TD-DFT level, from snapshots extracted from the $N P T$-MD simulations at constant time intervals of $500 \mathrm{ps}$. In each of the extracted snapshots, all the atoms of PMMA residues which had at least one atom within $15 \AA$

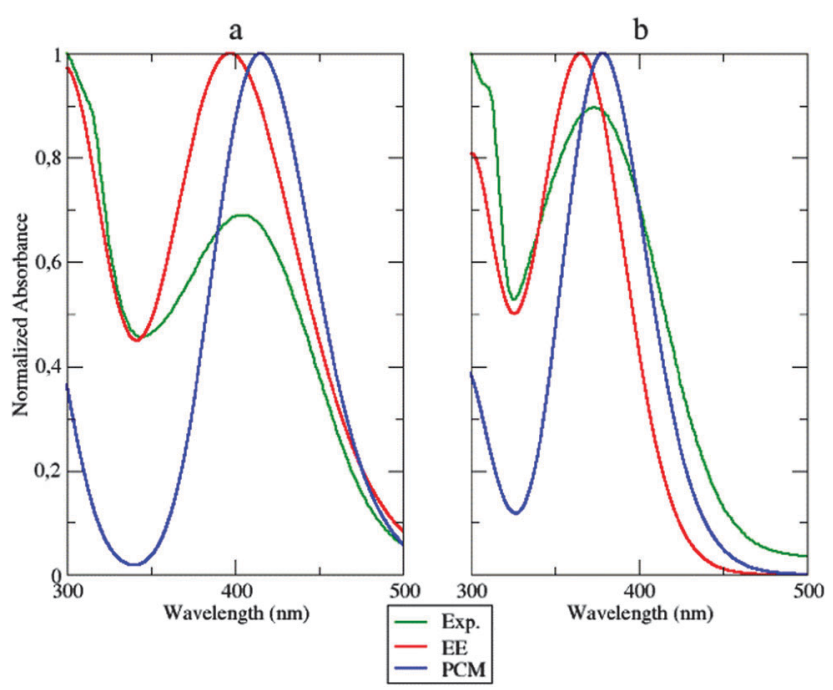

Fig. 11 Absorption spectra of $\mathbf{a}$ (left) and $\mathbf{b}$ (right panel) computed using the dynamic approach (EE, continuous red line) and the static approach (PCM, blue line), compared to the experimental spectrum (Exp., continuous green line). 
Table 1 Wavelength values calculated at the lie peak for both $\mathbf{a}$ and $\mathbf{b}$ upon dispersion in the PMMA matrix

\begin{tabular}{lll}
\hline System & Method & Absorption peak (nm) \\
\hline a in PMMA & Experimental & 404 \\
& Static approach (PCM) & 415 \\
& Dynamic approach (EE) & 397 \\
b in PMMA & Experimental & 373 \\
& Static approach (PCM) & 378 \\
& Dynamic approach (EE) & 365
\end{tabular}

from the fluorophore have been replaced by the respective atomic charge (according to the OPLS FF).

Computed UV absorption spectra for the fluorophore a are reported in Fig. 11, left panel.

Both the approaches mentioned above (the static and the dynamic one) were applied and compared with experiments. Inspection of Fig. 11 shows that both the static and dynamic approaches overestimate the absorbance intensity at the maximum absorption wavelength. Nonetheless, the dynamic approach reproduces very well the first peak at approximately $300 \mathrm{~nm}$, whose intensity is underestimated in the static model. This last approach shifts the absorption wavelength by about $11 \mathrm{~nm}$, while the same experimental peak is underestimated by $7 \mathrm{~nm}$ using the dynamic approach. The same observations could be extended to system $\mathbf{b}$ (Fig. 11, right panel). The overall shape of the experimental spectrum is again better reproduced by the dynamic simulation than by its static counterpart. The two approaches show, instead, comparable errors (of opposite sign) concerning the position of the peak maximum.

Computational and experimental findings are summarized in Table 1. Globally, inspection of the shape of the computed spectra evidences a good match with experiments, especially concerning the decay slope. Furthermore, the good agreement between the results obtained from atomistic and continuous descriptions of the environment suggests the absence of strong specific solute-solvent interactions (especially of electrostatic origin). Although the dynamic atomistic approach improves the reproduction of the spectral shape (especially in the area under $350 \mathrm{~nm}$ ), it is worth noticing that PCM performs a remarkable job in simulating the main features of the polymer embedding, permitting a preliminary semi-quantitative analysis of general trends by a fast approach.

\section{Conclusions}

Purposely tailored FFs have enabled us to follow the time evolution of two alkynylimidazole fluorophores when dispersed in a PMMA matrix intended to be part of a sunlight-harvesting device. Careful analysis of MD trajectories highlighted an overall rigidity of such hetero-aromatic molecular structures, due to the presence of the polymer chains, which hindered interconversions between different low-energy conformers. Analogous simulations in chloroform solution showed, instead, a significant flexibility of the dyes. These results confirm the hypothesis advanced recently to explain the different behavior of these dyes in solution and in the polymeric matrix.

Next, the attention was focused on the computation of absorption spectra of the same fluorophores within the polymer matrix. This was achieved by using two different methods, i.e. (i) a static approach, where spectroscopic computations were made only on the conformational minimum, modeling the environment using a polarizable continuum model (PCM), and (ii) a dynamic approach, where the same computations were made on several snapshots extracted from the MD trajectories, and explicitly representing the polymer through the so-called electrostatic embedding (EE). Even if a more correct reproduction of absorption spectra was achieved by the application of the second, more demanding, protocol, it has to be noticed that a static approach also works pretty well. In particular, the static protocol is confirmed in this work to be a very useful tool in virtual screening campaigns, where the best structures for the abovementioned applications have to be chosen by fast yet sufficiently accurate methods. It is, however, apparent that reliable optical features can be obtained using only force fields tailored for the correct reproduction of both structural and electrostatic (here by means of CM5 charges) properties.

The proposed approaches could also be extended to the simulation of emission spectra: the validity of the CAM-B3LYP theoretical scheme in the computation of emission properties is currently under investigation in our group, together with the parameterization of ad hoc FFs for the excited states of the two tested dyes, to be used during MD simulations in the dynamic approach. Future applications of the proposed procedures could also cover the inclusion of new, polar polymer matrices, as well as other condensed-phase environments, in order to further assess the validity of implicit and explicit models.

\section{Acknowledgements}

The research leading to the results had received funding from the European Union's Seventh Framework Programme (FP7/ 2007-2013) under grant agreement no. ERC-2012-AdG-320951DREAMS. The authors thank the DREAMS Lab technical staff for managing the computing facilities at SNS and Nicola De Mitri for useful discussions.

\section{References}

$1 \mathrm{~J}$. Harmsen and J. B. Powell, Sustainable Development in the Process Industries: Cases and Impact, John Wiley \& Sons, Inc., Hoboken, New Jersey, 2010.

2 K. E. Geckeler and R. Edward, Functional Nanomaterials, American Scientific Publisher, Stevenson Ranch, California, 2006.

3 X. D. Liu, A. R. Esker, M. Haeussler, C. Kim, P. Lucas, M. Matsunaga, N. Nishi, J. J. Robin, B. Z. Tang, D. A. Wang, M. Yamada and H. Yu, Functional Materials and Biomaterials, Adv. Polym. Sci., Springer-Verlag, Berlin, Heidelberg, 2007, vol. 209. 
4 F. Ciardelli, G. Ruggeri and A. Pucci, Chem. Soc. Rev., 2013, 42(3), 857-870.

5 S. K. Yesodha, C. K. S. Pillai and N. Tsutsumi, Prog. Polym. Sci., 2004, 29, 45-74.

6 G. S. Kumar and D. C. Neckers, Chem. Rev., 1989, 89, 1915-1925.

7 A. Natansohn and P. Rochon, Chem. Rev., 2002, 102, 4139-4176.

8 S. L. R. Barker, D. Ross, M. J. Tarlov, M. Gaitan and L. E. Locascio, Anal. Chem., 2000, 72, 5925-5929.

9 K. Komori, H. Matsui and T. Tatsuma, Bioelectrochemistry, 2005, 65, 129-134.

10 A. Pucci, G. Ruggeri and R. Bizzarri, Soft Matter, 2011, 7, 3689-3700.

11 J. Houghton, Global Warming, Cambridge University Press, 3rd edn, 2005.

12 W. G. van Sark, Renewable Energy, 2013, 49, 207-210.

13 M. G. Debije and P. P. C. Verbunt, Adv. Energy Mater., 2012, 2, 12-35.

14 F. Bures, $R S C A d v ., 2014$, 4, 58826-58849.

15 B. C. Rowan, L. R. Wilson and B. S. Richards, IEEE J. Sel. Top. Quantum Electron., 2008, 14, 1312-1322.

16 J. Bloino, J. Phys. Chem. A, 2015, 119, 5269-5287.

17 S. Fantacci, F. De Angelis, A. Sgamellotti, A. Marrone and N. Re, J. Am. Chem. Soc., 2005, 127, 14144-14145.

18 V. Barone, J. Bloino, S. Monti, A. Pedone and G. Prampolini, Phys. Chem. Chem. Phys., 2011, 13, 2160-2166.

19 G. Prampolini, F. Bellina, M. Biczysko, C. Cappelli, L. Carta, M. Lessi, A. Pucci, G. Ruggeri and V. Barone, Chem. - Eur. J., 2013, 19, 1996-2004.

20 N. A. Murugan, R. Apostolov, Z. Rinkevicius, J. Kongsted, E. Lindahl and H. Agren, J. Am. Chem. Soc., 2013, 135, 13590-13597.

21 N. De Mitri, G. Prampolini, S. Monti and V. Barone, Phys. Chem. Chem. Phys., 2014, 16, 16573-16587.

22 J. Tomasi, B. Mennucci and R. Cammi, Chem. Rev., 2005, 105(8), 2999-3094.

23 N. De Mitri, S. Monti, G. Prampolini and V. Barone, J. Chem. Theory Comput., 2013, 9, 4507-4516.

$24 \mathrm{M} . \mathrm{Pu}$ and T. Privalov, Chem. - Eur. J., 2015, 21, 17708-17720.

25 M. Sulpizi, P. Carloni, J. Hutter and U. Rothlisberger, Phys. Chem. Chem. Phys., 2003, 5, 4798-4805.

26 O. Crescenzi, M. Pavone, F. De Angelis and V. Barone, J. Phys. Chem. B, 2005, 109, 445-453.

27 N. A. Murugan, J. Phys. Chem. B, 2011, 115, 1056-1061.

28 V. Barone, F. Bellina, M. Biczysko, J. Bloino, T. Fornaro, C. Latouche, M. Lessi, G. Marianetti, P. Minei, A. Panattoni and A. Pucci, Phys. Chem. Chem. Phys., 2015, 17, 26710-26723.

29 O. B. Malcioglu, A. Calzolari, R. Gebauer, D. Varsano and S. Baroni, J. Am. Chem. Soc., 2011, 133, 15425-15433.

30 I. Cacelli and G. Prampolini, J. Chem. Theory Comput., 2007, 3, 1803-1817.
31 V. Barone, I. Cacelli, N. De Mitri, D. Licari, S. Monti and G. Prampolini, Phys. Chem. Chem. Phys., 2013, 15, 3736-3751.

32 F. Bellina, C. Calandri, S. Cauteruccio and R. Rossi, Tetrahedron, 2007, 63, 1970-1980.

33 F. Bellina, M. Lessi and C. Manzini, Eur. J. Org. Chem., 2013, 5621-5630.

34 F. Bellina, M. Lessi, G. Marianetti and A. Panattoni, Tetrahedron Lett., 2015, 56, 3855-3857.

35 K. Danel and L. J. T'suen, ARKIVOC, 2002, 1, 12-18.

36 A. V. Marenich, S. V. Jerome, C. J. Cramer and D. G. Truhlar, J. Chem. Theory Comput., 2012, 8, 527-541.

37 V. Barone, P. Cimino and E. Stendardo, J. Chem. Theory Comput., 2008, 4, 751-764.

38 V. Barone and P. Cimino, J. Chem. Theory Comput., 2009, 5, 192-199.

39 M. Cossi, G. Scalmani, N. Rega and V. Barone, J. Comput. Chem., 2003, 24, 669-681.

40 G. G. Hall and C. M. Smith, Int. J. Quantum Chem., 1984, 25, 881-890.

41 C. M. Smith and G. G. Hall, Theor. Chem. Acc., 1986, 69, 63-69.

42 W. L. Jorgensen, D. S. Maxwell and J. Tirado-Rives, J. Am. Chem. Soc., 1996, 118, 11225-11236.

43 M. J. Frisch, G. W. Trucks, H. B. Schlegel, G. E. Scuseria, M. A. Robb, J. R. Cheeseman, G. Scalmani, V. Barone, B. Mennucci, G. A. Petersson, H. Nakatsuji, M. Caricato, X. Li, H. P. Hratchian, J. Bloino, B. G. Janesko, A. F. Izmaylov, F. Lipparini, G. Zheng, J. L. Sonnenberg, W. Liang, M. Hada, M. Ehara, K. Toyota, R. Fukuda, J. Hasegawa, M. Ishida, T. Nakajima, Y. Honda, O. Kitao, H. Nakai, T. Vreven, K. Throssell, J. A. Montgomery, Jr., J. E. Peralta, F. Ogliaro, M. Bearpark, J. J. Heyd, E. Brothers, K. N. Kudin, V. N. Staroverov, T. Keith, R. Kobayashi, J. Normand, K. Raghavachari, A. Rendell, J. C. Burant, S. S. Iyengar, J. Tomasi, M. Cossi, N. Rega, J. M. Millam, M. Klene, J. E. Knox, J. B. Cross, V. Bakken, C. Adamo, J. Jaramillo, R. Gomperts, R. E. Stratmann, O. Yazyev, A. J. Austin, R. Cammi, C. Pomelli, J. W. Ochterski, R. L. Martin, K. Morokuma, V. G. Zakrzewski, G. A. Voth, P. Salvador, J. J. Dannenberg, S. Dapprich, P. V. Parandekar, N. J. Mayhall, A. D. Daniels, O. Farkas, J. B. Foresman, J. V. Ortiz, J. Cioslowski and D. J. Fox, Gaussian Development Version, Revision I.03, Gaussian, Inc., Wallingford, CT, 2014. 44 J. Wang, R. M. Wolf, J. W. Caldwell, P. A. Kollman and D. A. Case, J. Comput. Chem., 2004, 25(9), 1157-1174.

45 H. J. C. Berendsen, J. P. M. Postma, W. van Gunsteren, A. Di Nola and J. R. Haak, J. Chem. Phys., 1984, 81, 3684-3690.

46 http://virtualchemistry.org/, accessed December 2015.

47 B. Hess, H. Bekker, H. J. C. Berendsen and J. G. E. M. Fraaije, J. Comput. Chem., 1997, 18, 1463-1472.

48 D. van der Spoel, E. Lindahl, B. Hess and GROMACS development team, GROMACS User Manual version 4.6.5, 2013, www.gromacs.org. 